\title{
miR-519d-3p Overexpression Inhibits P38 and PI3K/AKT Pathway via Targeting VEGFA to Attenuate the Malignant Biological Behavior of Non-Small Cell Lung Cancer
}

This article was published in the following Dove Press journal: OncoTargets and Therapy

\author{
Guangzhao Zhang ${ }^{1} *$ \\ Yanlei $\mathrm{Hu}^{2, *}$ \\ Wuying Yuan' \\ Hongli Qiu' \\ Haifeng Yu' \\ Jiahui $\mathrm{Du}{ }^{\prime}$ \\ 'Department of Minimally Invasive \\ Surgery, Henan Chest Hospital, \\ Zhengzhou, Henan 450003, People's \\ Republic of China; ${ }^{2}$ Department of \\ Cardiovascular Surgery, Fuwai Central \\ China Cardiovascular Hospital, \\ Zhengzhou, Henan 45I464, People's \\ Republic of China \\ *These authors contributed equally to \\ this work
}

\begin{abstract}
Background: Non-small cell lung cancer (NSCLC) is a heterogeneous tumor that accounts for approximately $85 \%$ of all lung cancer cases worldwide. microRNAs (miRNAs) are believed to play an important role in regulating a variety of biological processes, including immunity and cancer. We investigated the effect of miR-519d-3p on the mitigation of NSCLC in vitro and in vivo.

Methods: RT-PCR or immunohistochemical assays were used to assess the expression of miR-519d-3p. Colony formation, flow cytometry, and transwell assay were respectively used to detect proliferation, apoptosis, and invasion of A549 and NCI-H661 cell lines. Luciferase reporter assay was used to verify targeting the relationship between mir-519d-3p and VEGFA. Western blot was used to examine the expression of Ki67, caspase-3, E-cadherin, $\mathrm{N}$-cadherin, VEGF, P38, and PI3K/AKT. Animal models were established by BABL/c mice to research the effect of mir-519d-3p overexpression in vivo.
\end{abstract}

Results: In vitro, miR-519d-3p overexpression inhibited A549 and NCI-H661 cells proliferation, invasion, and also promoted apoptosis. In addition, miR-519d-3p overexpression downregulated VEGFA expression and decreased the P38 and PI3K/AKT phosphorylation level. In vivo, miR-519d-3p overexpression significantly restrained tumor volume (2087 $\pm 265 \mathrm{~mm}^{3}$ vs $\left.599 \pm 135 \mathrm{~mm}^{3}, * P<0.05\right)$ and tumor weight $(0.45 \pm 0.08 \mathrm{~g}$ vs $0.13 \pm 0.06 \mathrm{~g}$, $* P<0.05)$ compared with the control group. Overexpression of miR-519d-3p downregulated levels of Ki67 and N-cadherin significantly.

Conclusion: The data indicated that miR-519d-3p could be a novel therapy or adjuvant against NSCLC.

Keywords: miR-519d-3p, invasion, non-small cell lung cancer, A549 cells, apoptosis

\section{Introduction}

Currently, the prominent reason of cancer-related death in the world is lung cancer. The total number of lung cancer deaths accounted for about $23 \%$ of total cancer deaths in 2015 in China. ${ }^{1}$ Approximately $80 \%$ of lung cancers are classified as nonsmall cell lung cancer (NSCLC) and are clinically and pathologically different from small cell lung cancer (SCLC). ${ }^{2}$ NSCLC is a heterogeneous tumor that accounts for about $85 \%$ of all lung cancer cases worldwide. ${ }^{3}$ The reasons for these phenomena are usually due to the later appearance of lung cancer and the lack of treatment options. ${ }^{4}$ Where feasible, surgical resection is still the most consistent and 
successful treatment. Nevertheless, nearly $70 \%$ of lung cancer patients have local advanced or metastatic disease at the time of diagnosis. ${ }^{4}$ Therefore, it is urgent to find new drugs and therapeutic strategies. In recent years, targeted therapy has emerged as an important mean of the disease management for NSCLC. ${ }^{5}$

MicroRNAs (miRNAs) are endogenous non-coding RNAs with about 22 nucleotides. They regulate gene expression by binding to the $3^{\prime}$ untranslated region ( $3^{\prime}$ UTR) of mRNAs, thus inhibiting the translation or induction of mRNA disruption. ${ }^{6}$ miRNAs have been deemed to be critical in the regulation of a variety of biology, including immunity and cancer. ${ }^{7,8}$ In recent years, researchon miRNA regulating NSCLC development has been fruitful. Shantanu et $\mathrm{al}^{8}$ reported that miR-34a and miR-16 can coordinately control the cell cycle checkpoint in NSCLC cells to intervene in cell fate mechanisms of NSCLC cells. Articles reports that miR-519 was involved in the regulation of lung cancer processes. ${ }^{9,}{ }^{10}$ Cheng et al ${ }^{11}$ reported that knockdown of LINC01419 inhibited cell proliferation and metastasis in lung adenocarcinoma via upregulating miR-519b-3p.

Tumor growth requires angiogenesis to remodel blood vessels and further to provide a pathway for metastatic tumor cells. ${ }^{12}$ Vascular endothelial growth factor (VEGF), highly expressed in cancers, plays an important role in angiogenesis. VEGF was considered as an attractive therapeutic target for anti-angiogenic therapy. VEGF is a member of the platelet-derived growth factor family, including VEGFA, VEGFB, VEGFC, VEGFD, and VEGFE. ${ }^{13}$ Upregulation of the VEGFA was deemed to be a poor prognostic factor for tumor-free survival of osteosarcoma. ${ }^{14}$ Hence, VEGFA can be regarded as a potential target of cancer therapy. Meade et $\mathrm{al}^{15}$ reported that VEGFA strengthened coexpression of the inhibitory receptor TIM-3 on T cells and the combined use of antiPD-L1 and anti-VEGF therapy may be an effective treatment strategy for SCLC patients. Reports suggested that VEGF functioned through the PI3K/Akt and p38 MAPK signaling pathways in some cancer development processes. For example, VEGFA/VEGF 2 axis accelerates the migration of dental pulp stem cell through the p38 MAPK and FAK/PI3K/Akt signaling pathways. ${ }^{16}$

Despite miR-519 being involved in lung cancer regulation, it is not clear whether miR-519 plays an inhibitory role through VEGFA and PI3K/AKT and P38 pathways. In this study, A549 and NCI-H661 cells were used as experimental cell lines to investigate the anti-tumor effect of
miR-519d-3p on lung cancer in vitro. Of course, experiments in vivo were conducted on transplanted tumor models which were set up by $\mathrm{BABL} / \mathrm{c}$ female nude mice.

\section{Materials and Methods Cell Culture}

The lung cell lines A549 and NCI-H661 cells were purchased from American Type Culture Collection (ATCC, Manassas, VA). A549 cells were cultured in F12 Kaighn's Medium (Invitrogen, Carlsbad, CA) with 10\% FBS, 100 $\mathrm{U} / \mathrm{mL}$ penicillin, and $100 \mathrm{U} / \mathrm{mL}$ streptomycin under standard culture conditions and activated for 2-3 passages. NCI-H661 was cultured in RPMI 1640 (Invitrogen) with the same conditions. The cultures were incubated at $37^{\circ} \mathrm{C}$ in $5 \% \mathrm{CO}_{2}$ under $95 \%$ humidified air.

\section{Cell Transfection}

miR-519d-3p mimics, miR-519d-3p inhibitor, pcDNA VEGFA, and shVEGFA were designed and synthesized by Molbase biological (Henan, China). Upon PCR amplification, pcDNA VEGFA was inserted into the pcDNA3.1 plasmid. A549 and NCI-H661 cells were respectively inoculated into 6-well plates. As $70-80 \%$ fusion, the cells were transfected with mimics, inhibitor, pcDNA VEGFA, and shVEGFA (Molbase, Henan, China), respectively. After 6 hours of transfection, the fresh medium was replaced. Forty-eight hours after transfection, the cells were harvested. RT-PCR and Western blot were used to confirm the transfection efficiency.

Groups: the control group, mimic-NC group, miR519d-3p mimic group, miR-519d-3p inhibitor group, pcDNA group, shVEGFA group, pcDNA-VEGFA group, miR-519d-3p mimic + pcDNA-VEGFA (mimic + VEGFA) group.

\section{Colony Formation Assay}

NCI-H661 and $5 \times 10^{3}$ A549 cells were seeded into 6-well plates overnight to detect colony formation. After being in culture for 10 days, visible cells were washed with phosphate-buffered saline (PBS), fixed with methanol, and stained with Giemsa. Finally, the colonies about 50 cells were counted.

\section{Transwell Assay}

After being diluted with serum-free medium, matrigel was applied to the bottom of the superior cavity and dried at room temperature for later use. After incubation in serum- 
free medium for 24 hours, the cell suspension was added to the upper chamber ( $200 \mathrm{~mL}$ each chamber). The lower chambers were filled with $600 \mu \mathrm{L}$ medium containing $10 \%$ FBS. After incubation at $37^{\circ} \mathrm{C}$ for 24 hours, the incubator was taken out and washed twice with PBS. The residual cells were removed by a cotton bud. The cells were fixed with 95\% ethanol and stained with crystal violet. The average number of invasive cells was recorded by microscope (Leica, Germany).

\section{Flow Cytometry}

Upon 48 hours transfection, cells were harvested to detect cell apoptosis. The cells were washed three times with precooled PBS, and then resuspended with a binding buffer of $1 \times 10^{6} / \mathrm{mL}$ to $5 \times 10^{6} / \mathrm{mL}$. Next, $100 \mu \mathrm{L}$ suspension was transferred into a $5-\mathrm{mL}$ test tube, to which $5 \mu \mathrm{L}$ of Annexin V/ fluorescein isothiocyanate (FITC) and $5 \mu \mathrm{L}$ of propidium iodide (PI) were added, and the tube was incubated in the dark for 15 minutes. Then, $400 \mu \mathrm{L}$ of $1 \times$ Annexin $\mathrm{V}$ binding buffer was added into the tube. Cell apoptosis was analyzed using a FACScan flow cytometer (BD Biosciences, USA).

\section{RT-PCR}

The total RNAs were extracted using the TRIzolTM kit (Invitrogen; Waltham, MA, USA). Upon quantification, the cDNA synthesis Kit (Invitrogen) was used to synthesize cDNA with $0.5 \mu \mathrm{g}$ RNA from each group. MiR-519d$3 p$ and VEGFA levels were measured by SYBR PCR MasterMix kit (Invitrogen). The GAPDH was treated as an internal reference to standardize expression of the tested gene following the $2^{-\Delta \Delta \mathrm{Ct}}$ method. ${ }^{17}$

\section{Luciferase Reporter Assay}

TargetScan software showed that VEGFA and miR519d-3p have a good targeting relationship. The wildtype (wt) fragments from VEGFA (VEGFA-wt) with the potential binding sites and mutant type (mut) fragments from VEGFA (VEGFA-mut) and miR-519d-3p were inserted into the pMIR-report plasmid vector (Promega, USA) to generate the VEGFA luciferase reporter construct. Next, the miR-519d-3p mimic and vectors were co-transfected into A549 cells using Lipofectamine 2000 reagent (Invitrogen, Carlsbad, CA, USA). ${ }^{18}$ Upon transfection for 24 hours, the cells were detected using a luciferase detection kit (Promega). The ratio of Rluc/ Luc was recorded.

\section{Tumor Formation Assay}

Four week old BABL/C female nude mice were used to establish the animal model. None of the mice had a specific pathogen. A549 cells transfected with mir519d-3p lentivirus or empty vector were injected into the right dorsal side of $\mathrm{BABL} / \mathrm{C}$ nude mice. The volume of tumor was detected every 5 days. The animals were killed at 30 days after injection, the transplanted tumor was dissected and weighed, and RT-PCR and immunohistochemistry were performed. All the animal experiments were approved by the Institutional Animal Care and Use Committee of the Henan Chest Hospital and were conducted strictly in accordance with the guidelines of International Guiding Principles for Animal Research.

\section{Immunohistochemistry (IHC)}

The IHC analysis was operated as previously reported. ${ }^{19}$ Tumor tissue was fixed with 4\% formalin and embedded with paraffin. The tissue embedded with paraffin was cut into $3 \mu \mathrm{m}$ thick sections. Then, the sections were deparaffinized in xylene and rehydrated in an alcohol gradient routinely. Tissue antigen was retrieved by heating in sodium citrate $(\mathrm{pH}$ 6.0) for 10 minutes and sections were sealed with 5\% normal goat serum for 1 hour and co-incubation with primary antibodies Ki67 (ab15580, 1:500), N-cadherin (ab76011, 1:1000) overnight at $4^{\circ} \mathrm{C}$. After been washed three times in PBS, the sections were incubated with secondary antibody (ZSGB-BIO, Beijing, China) for 30 minutes at room temperature. Then, sections were stained using a SignalStain DAB Substrate Kit (Invitrogen; Waltham, MA, USA) and analyzed by Olympus BX-UCB light-field microscope (Shinjuku, Tokyo, Japan).

\section{Western Blot Analysis}

Western blot analysis was operated following the previous report. ${ }^{20}$ Briefly, tissues $(0.1 \mathrm{~g})$ were homogenized in $1 \mathrm{~mL}$ protein extraction buffer. Then, centrifugation and collected supernatant detected the protein with a BCA assay kit. The total protein samples $(20 \mu \mathrm{g})$ were put into $10 \%$ sodium dodecyl sulfate polyacrylamide gel electrophoresis (SDS PAGE) loading buffer and afterwards were transferred to polyvinylidene difluoride (PVDF) membranes. The membranes were sealed up in 5\% skimmed milk for 120 minutes at $37^{\circ} \mathrm{C}$. Then, the following primary antibodies were incubated overnight at $4^{\circ} \mathrm{C}$ : caspase-3 (ab13847, 1:500), Ki-67 (ab15580, 1:500), N-cadherin (ab18203, 1:300), E-cadherin (ab1416, 1:50), VEGFA (ab52917, 1:1000), P38MAPK 
(ab31828, 1:1000), PI3K (ab140307, 1:2000), and AKT (ab8805, 1:500). The protein levels were analyzed by ImageJ software.

\section{Statistical Analysis}

Analyses were performed using SPSS 21.0 (SPSS, Inc, Chicago, IL, USA). One-way ANOVA was performed to assess multiple sets of data, as indicated. Data were presented as the mean $\pm \mathrm{SD}$. The difference was statistically significant when $P<0.05$.

\section{Results}

\section{Overexpression of miR-519d-3p Inhibits} the Growth of A549 and $\mathrm{NCl}-\mathrm{H} 66 \mathrm{I}$

From Figure 1A, miR-519d-3p successfully overexpressed in A549 and NCI-H661 cells. The introduction of miR519d-3p mimics significantly increased the expression of mir-519d-3p (Figure 1A). It indicated that stable lung cancer cell lines transfected with miR-519d-3p was set up.

Compared with the control group, colony the formation rate of miR-519d-3p mimic group was inhibited significantly in A549 and NCI-H661 cells (Figure 1B and C). As shown in Figure 1D and E, overexpression of miR-519d-3p markedly accelerated cell apoptosis. From Figure 1F, miR519d-3p overexpression markedly decreased Ki67 level in A549 and NCI-H661, with the increasing of cleaved cas3/ cas3. These findings indicate that miR-519d-3p overexpression has negative effects on colony formation, proliferation, and apoptosis of A549 and NCI-H661.

\section{Overexpression of miR-519d-3p Inhibits the Invasion of $\mathrm{A} 549$ and $\mathrm{NCl}-\mathrm{H} 66 \mathrm{I}$}

From Figure 2A and B, overexpression of miR-519d-3p markedly lessened the invasive cells number. In addition, overexpression of miR-519d-3p markedly upregulated E-cadherin level in A549 and NCI-H661 accompanied with significant decreasing of N-cadherin level (Figure 2C). These findings indicate that miR-519d-3p overexpression inhibited invasion of A549 and NCI-H661.

\section{miR-519d-3p Suppresses the}

Proliferation, Invasion, and Promotes Apoptosis of A549 by Targeting VEGFA

Since miR-519d-3p expression is negatively correlated with the malignant biological behavior of lung cancer cells, we used loss-of-function experiments to confirm whether it influences proliferation, migration, and apoptosis of A549 cells by targeting VEGFA. Western blot assays and RT-PCR were used to evaluate VEDFA expression in A549 cells. The results showed that VEDFA expression was decreased or increased after A549 cells were transfected with miR-519d-3p mimic or inhibitor, respectively (Figure 3A and B). Results of RT-PCR assay revealed that VEGFA was successfully knocked down in A549 cells (Figure 3C). Knockdown of VEGFA results in marked upregulation of miR-519d-3p, whereas its overexpression led to the miR-519d-3p downregulation (Figure 3D). TargetScan search software further predicts that miR-519d-3p can bind to the 3'-UTR region of VEGFA directly (Figure 3E). In order to obtain direct evidence of the interaction between VEGFA and miR$519 \mathrm{~d}-3 \mathrm{p}$, we subcloned the VEGFA-wt, VEGFA-mut, and miR-519d-3p binding site into dual-luciferase reporters. From Figure 3F, the relative luciferase activity of VEGFA-wt in A549 cells was evidently decreased after co-transfection of miR-519d-3p mimic, while it did not change mutant vector activation, which indicated that miR-519d-3p is a direct target of VEGFA. As expected, compared with the miR-519d-3p mimic group, the excellent colony-forming ability of VEGFA was inhibited in the miR-519d-3p mimic and pcDNA-VEGFA co-transfection groups (Figure 3G). Consistently, compared with the miR$519 \mathrm{~d}-3 \mathrm{p}$ mimic group, the group co-transfected with VEGFA and miR-519d-3p mimic also inhibited cell invasion ability and promoted apoptosis (Figure $3 \mathrm{H}$ and $\mathrm{I}$ ). These findings indicate that miR-519d-3p inhibits the proliferation and invasion, and promotes apoptosis of A549 by targeting VEGFA.

\section{miR-519d-3p Inhibits P38 and PI3K/AKT} Pathway

In this part, the activation of the $\mathrm{P} 38$ and PI3K/AKT pathway were detected by Western blot assay. From Figure 4A, compared with the control group, the phosphorylation of P38MAPK, PI3K, and AKT were significantly inhibited in the miR-519d-3p mimic group. In addition, the ratios of p-P38MAPK/P38MAPK, p-PI3K/ $\mathrm{PI} 3 \mathrm{~K}$, and $\mathrm{p}-\mathrm{AKT} / \mathrm{AKT}$ in the miR-519d-3p mimic and pcDNA-VEGFA co-transfection groups were significantly reduced compared with the miR-519d-3p mimic group (Figure 4B-D). These findings suggest that miR-519d-3p inhibits the P38 and PI3K/AKT pathway by targeting VEGFA. 
A

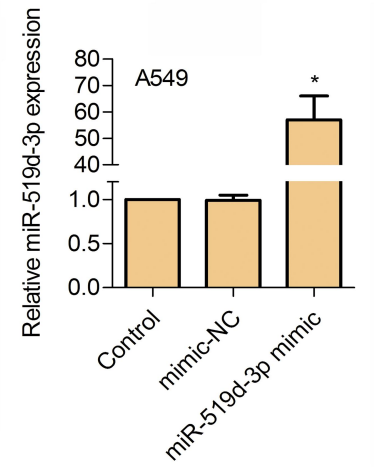

B

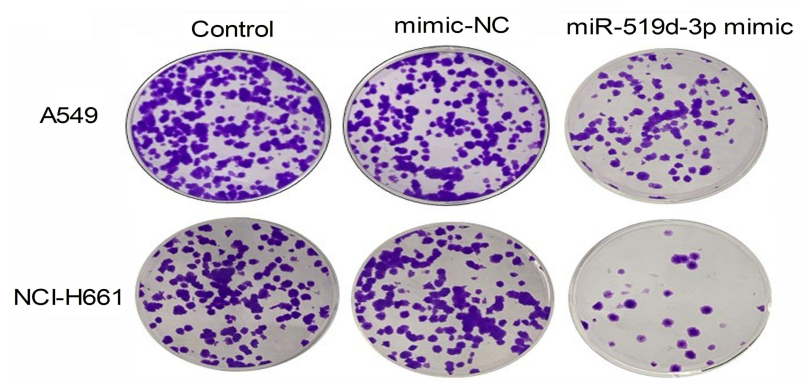

D
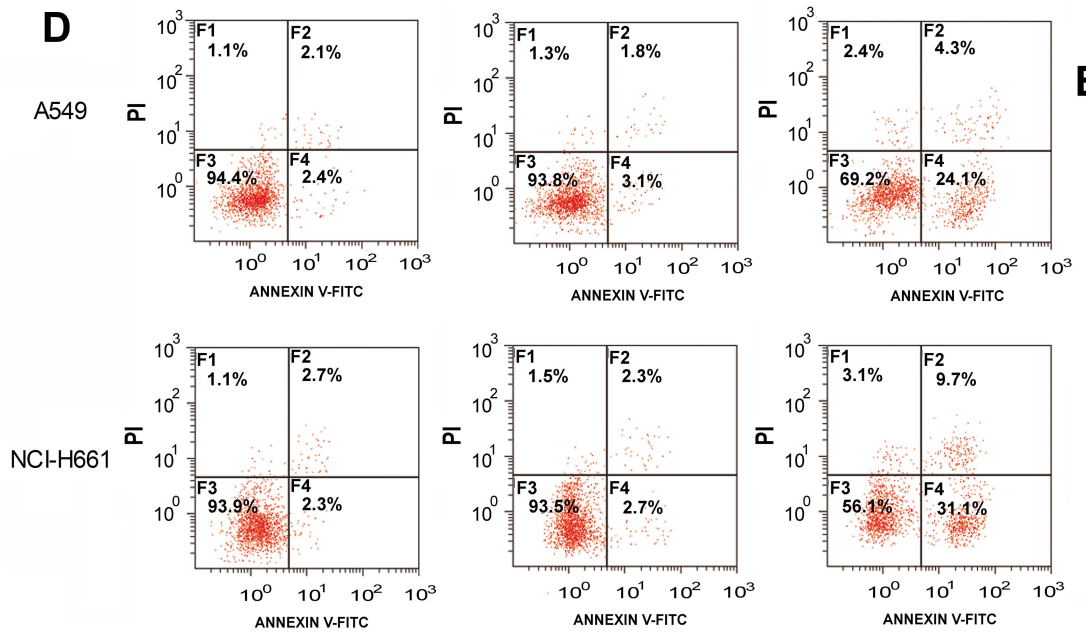

A549

F

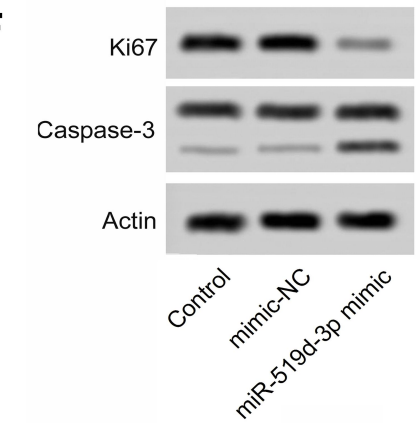

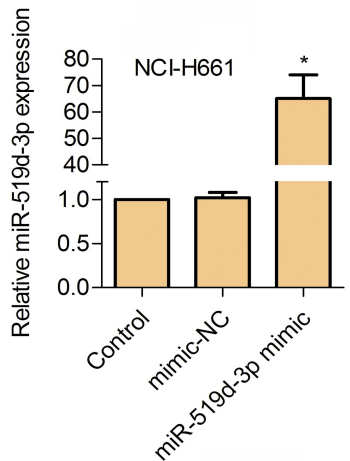

NCl-H661

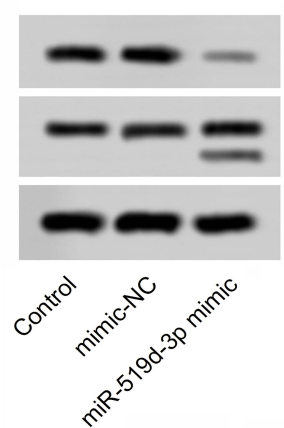

C
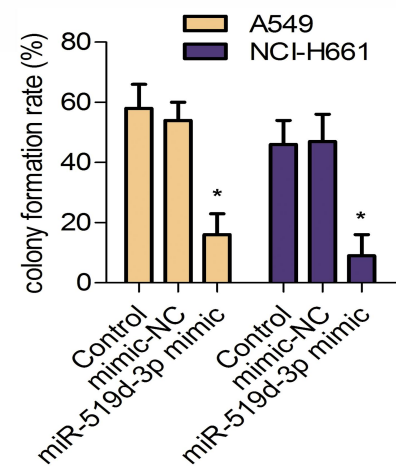

E
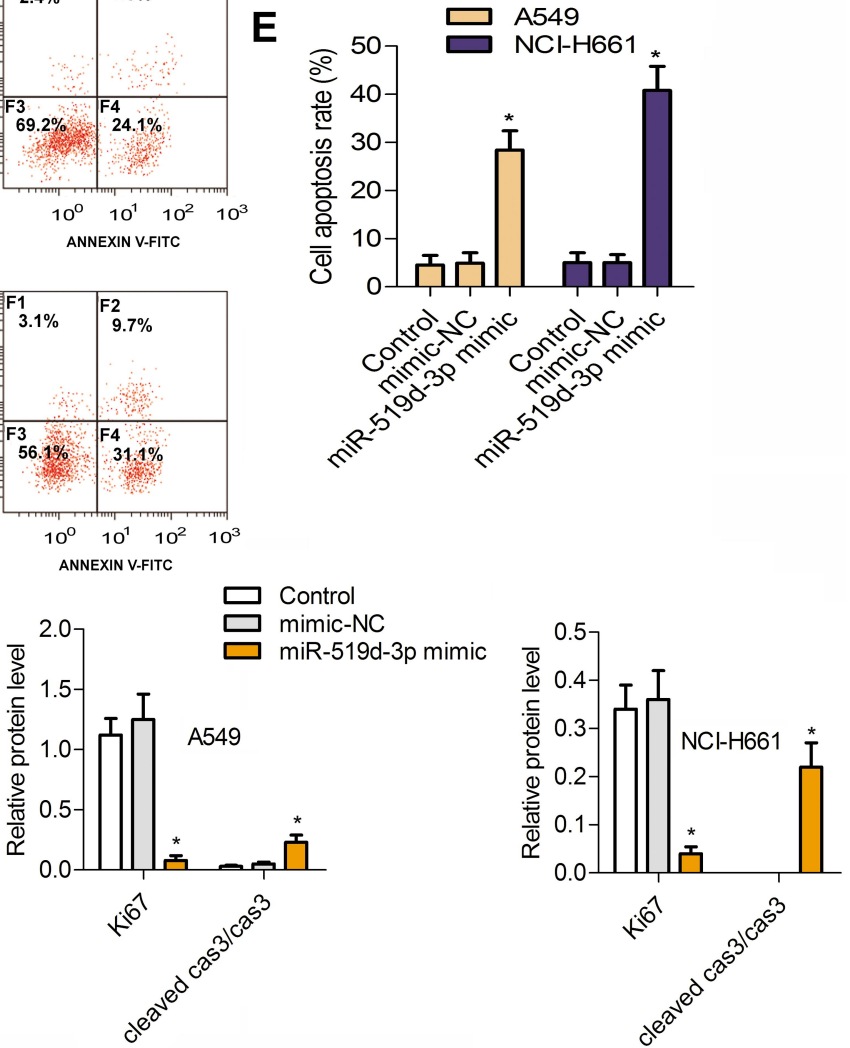

Figure I Overexpression of miR-5I9d-3p inhibits the growth of lung cancer cells A549 and NCl-H66I. (A) Establishment of lung cancer cell lines with miR-5I9d-3p overexpression. (B) Typical image of colony formation assay. (C) Percentage of colony formation rate. (D) Typical image of flow cytometry. (E) Percentage of colony formation rate cell apoptosis rate. (F) MiR-5I9d-3p overexpression inhibited the proliferation of lung cancer cells $\mathrm{A} 549$ and $\mathrm{NCl}-\mathrm{H} 66 \mathrm{I}$ (*P<0.05 vs control). 
A

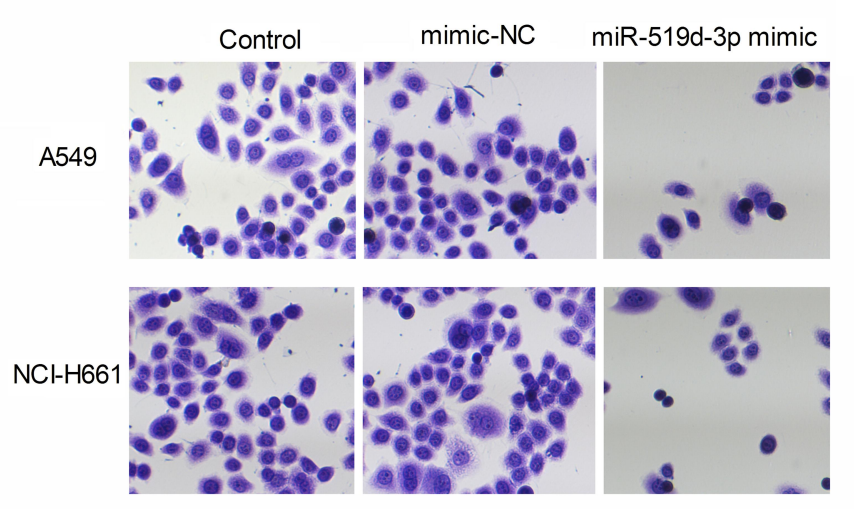

B

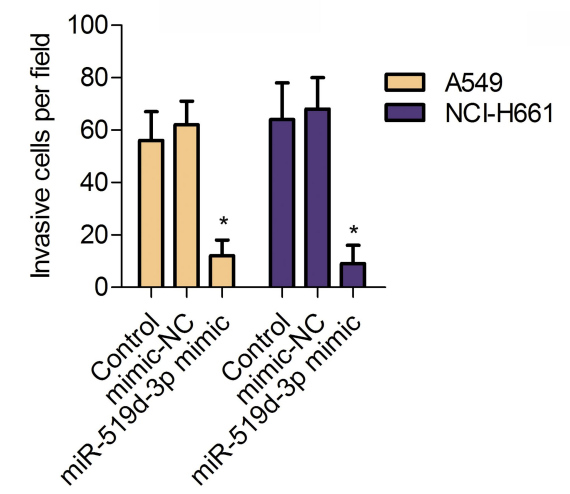

C
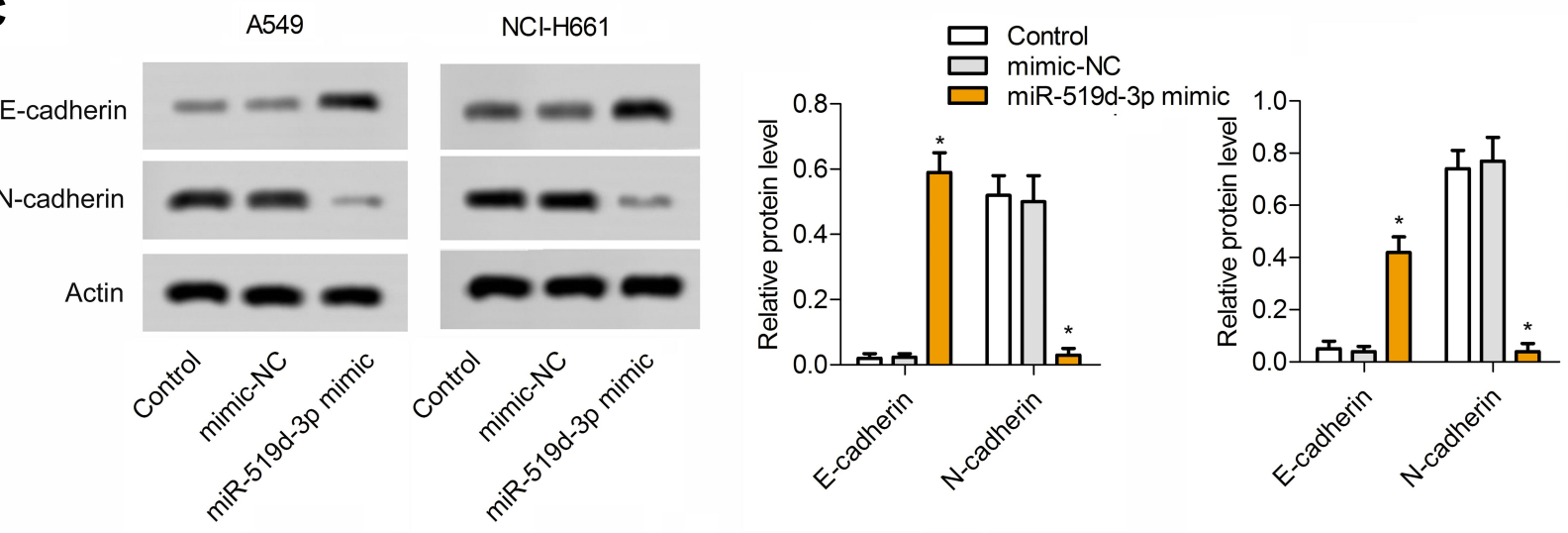

Figure 2 Overexpression of miR-519d-3p inhibits the invasion of lung cancer cells A549 and NCl-H66I. (A) Typical image of transwell assay. (B) Invasive cells per field. (C) MiR-519d-3p overexpression increased the expression of E-cadherin and decreased the expression of $\mathrm{N}$-cadherin in lung cancer cells $\mathrm{A} 549$ and $\mathrm{NCl}-\mathrm{H} 66 \mathrm{I}$. Data are expressed as mean $\pm S D$ of three independent experiments ( $* P<0.05$ vs control).

\section{miR-519d-3p Affects Tumor Formation in vivo}

In our study, the tumor model was established by female nude mice. Obviously, compared with the control group, transplanted tumors in the miR-519d-3p group were smaller in volume and lighter in weight (Figure 5A-C). Immunohistochemical results showed that the percentage of Ki67 and N-cadherin positive cells in the miR-519d-3p group were significantly higher than the control group (Figure 5D-F). These findings indicate that miR-519d-3p overexpression inhibits tumor formation in vivo.

\section{Discussion}

Lung cancer is still the most commonly diagnosed cancer and the main cause of cancer death in the world. Among all lung cancers, NSCLC accounts for $80-85 \%$, and most patients have locally advanced or metastatic disease at first diagnosis. ${ }^{4}$ The progressof lung cancer is rapid, and multiple metastases occur in advanced stages. Tumor metastasis requires multiple molecular mechanisms that trigger cancer cells, including angiogenesis, vascular remodeling, and immune escape. MirRNA has been extensively used in the diagnosis and treatment of lung cancer. ${ }^{21-23}$ The results of this study revealed that overexpressed miR-519d-3p inhibited tumor growth and progression by modulating angiogenesis via targeting VEGF. It is well known that angiogenesis is an important process of tumor cell proliferation and viability. Blocking VEGF/VEGFR signaling can destroy vascular supply and make tumor lack of nutrition and oxygen. ${ }^{24}$ In addition, research has shown that the VEGFA/VEGF receptor 2 axis exerted function via the FAK/PI3K/Akt and p38 MAPK signaling pathways. In this study, miR-519d-3p overexpression suppressed VEGFA expression and further inhibited the P38 and PI3K/AKT pathway to fade the malignant biological behavior of lung cancer. Stated thus, miR-519d-3p can be a novel treatment for lung cancer.

The regulation of mir-519d-3p is considered to be a potential strategy for the treatment of a variety of 


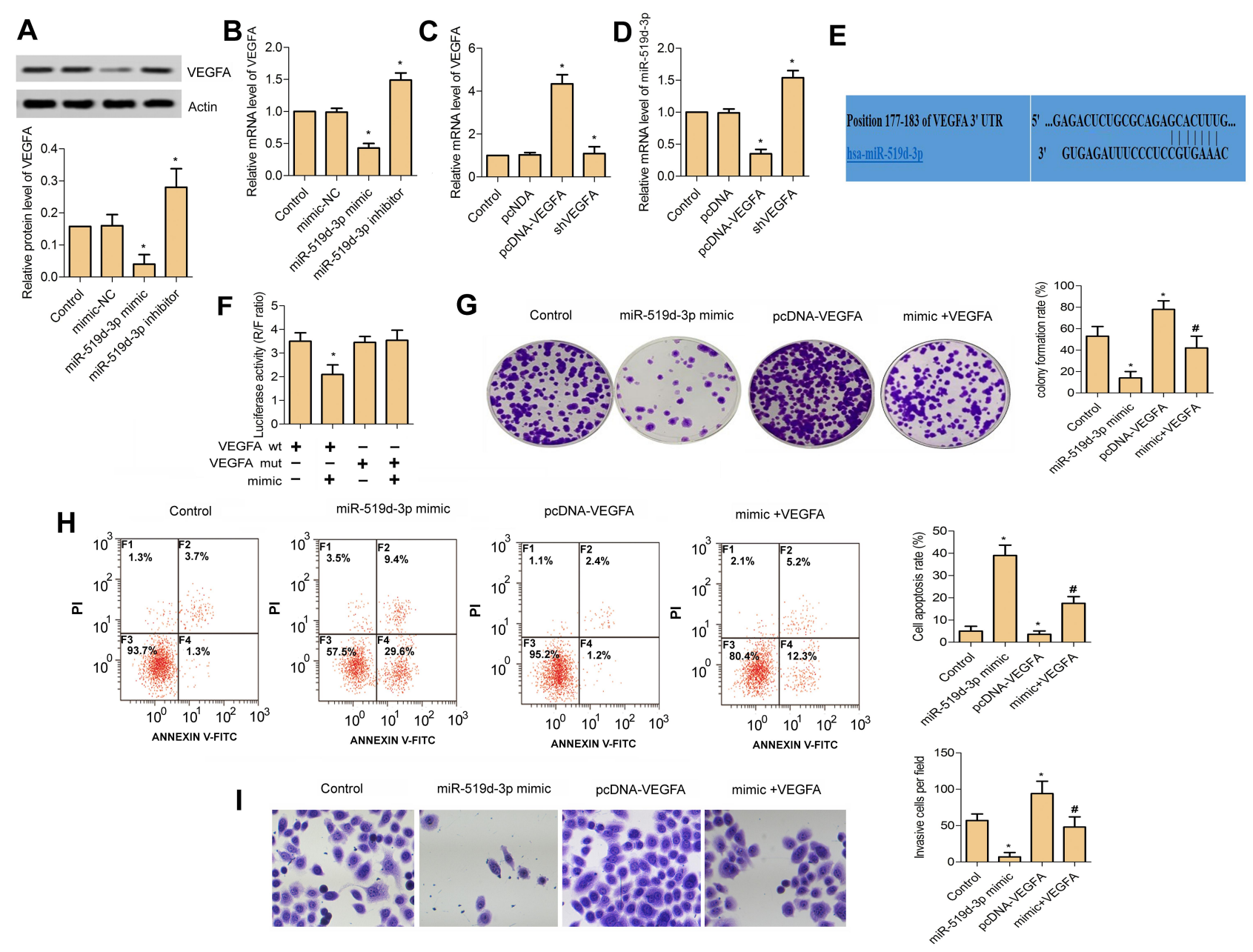

Figure 3 MiR-519d-3p inhibits the proliferation and invasion, and promotes apoptosis of lung cancer cell A549 by targeting VEGFA. (A) Relative protein level of VEGFA in A549 cell transfected with miR-519d-3p mimic and inhibitor. (B) Relative mRNA level of VEGFA in A549 cell transfected with miR-519d-3p mimic and inhibitor. (C) Relative mRNA level of VEGFA in A549 cell transfected with pcDNA-VEGFA and shVEGFA. (D) Relative mRNA level of miR-5I9d-3p in A549 cell transfected with pcDNA-VEGFA and shVEGFA. (E) TargetScan search software predicts that miR-519d-3p can directly bind to the 3'-UTR region of VEGFA gene. (F) Luciferase activity (R/F ratio). (G) Colony formation assay. (H) Flow cytometry. (I) Transwell assay ( ${ }^{* P}<0.05$ vs control; ${ }^{\#} P<0.05$ vs miR-5I9d-3p mimic).

cancers. Ma and $\mathrm{Li}^{25}$ reported that miR-519d-3p expression was significantly reduced in glioma cell lines and tissues, while overexpression of miR-519d-3p inhibited cell proliferation and induced cell cycle G0/G1 arrest. Liang et $\mathrm{al}^{26}$ reported that miR-591d-3p expressed lowly in pancreatic cancer cell lines and tissues, and overexpression of miR-519d-3p significantly inhibited the proliferation of pancreatic cancer cells and $\mathrm{Wnt} / \beta$-catenin signaling via targeting RPS15A. Ye and $\mathrm{Lv}^{17}$ reported that miR$519 \mathrm{~d}-3 p$ inhibited the proliferation and migration of colorectal cancer cells through targeting TROAP. In the present study, miR-519d-3p was expressed lowly in A549 and NCI-H661. Compared with the control group, miR-519d$3 p$ overexpression suppressed Ki67 expression and colony formation rate in the miR-519d-3p mimic transfected group of A549 and NCI-H661 significantly.
Overexpression of miR-519d-3p in A549 and NCIH661 elevated cleaved cas3/cas3 and expedited cell apoptosis significantly. Simultaneously, overexpression of miR-519d-3p enhanced E-cadherin expression significantly accompanied with significant decreasing of $\mathrm{N}$-cadherin expression in A549 and NCI-H661 cells. Overall, our findings suggested that miR-519d-3p overexpression suppressed cell proliferation, invasion, and expedited apoptosis of A549 and NCI-H661 cells.

VEGF is a highly specific pro-vascular endothelial growth factor that promotes increased vascular permeability, extracellular matrix degeneration, vascular endothelial cell migration, proliferation, and angiogenesis. VEGFA and VEGFR2 are critical molecules for angiogenesis. ${ }^{27}$

Using mirRNAs to regulate VEGF to inhibit angiogenesis and further to block cancer development has become 


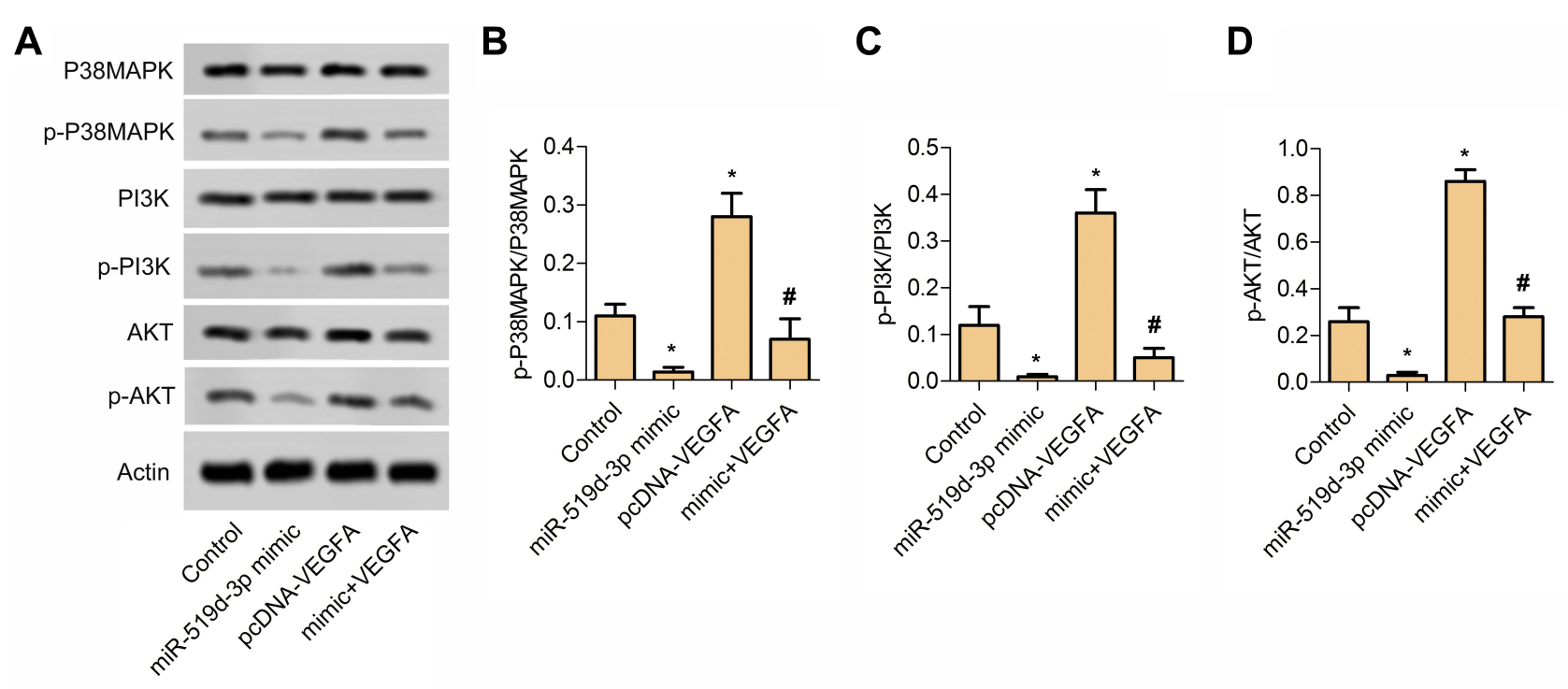

Figure 4 MiR-519d-3p inhibits P38 and PI3K/AKT pathway. (A) Typical image of Western blot assay. (B) The ratio of p-P38MAPK and P38MAPK. (C) The ratio of p-PI3K and PI3K. (D) The ratio of $\mathrm{p}-\mathrm{AKT}$ and AKT. Data are expressed as mean \pm SD of three independent experiments $\left({ }^{*} P<0.05\right.$ vs control; ${ }^{*} P<0.05$ vs miR-5I9d-3P mimic).

A

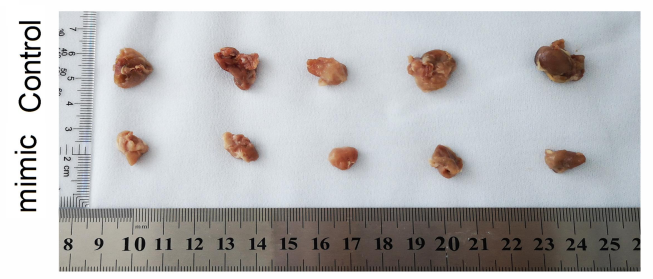

B

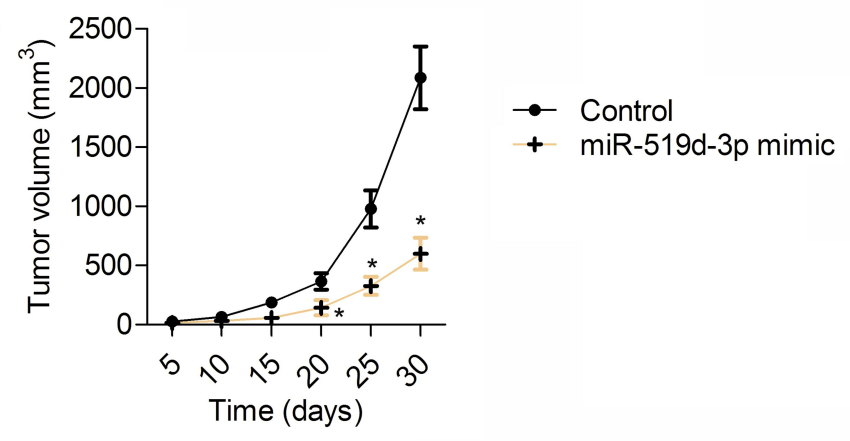

C

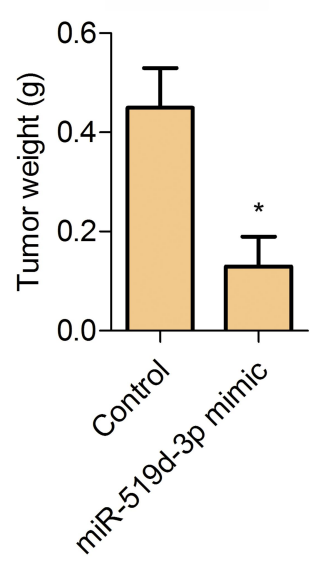

D

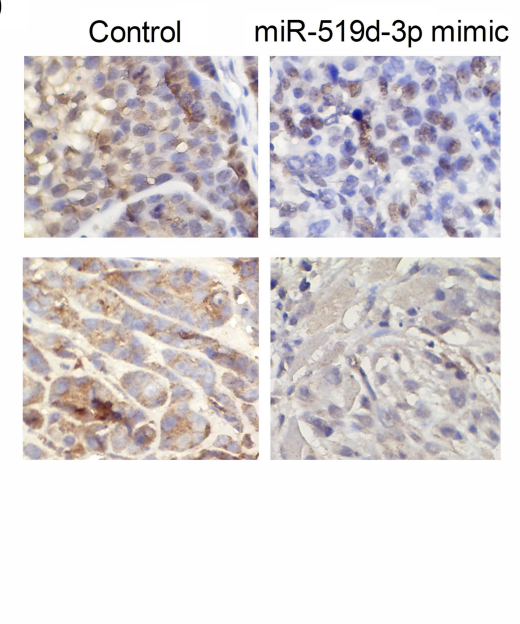

E

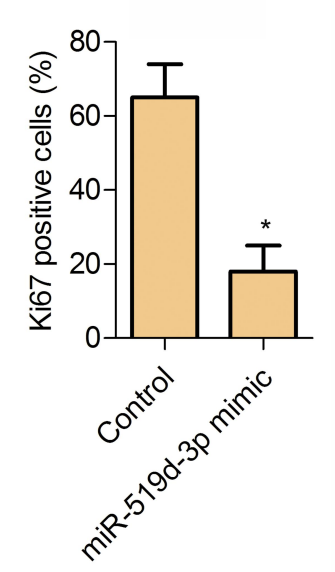

$\mathbf{F}$

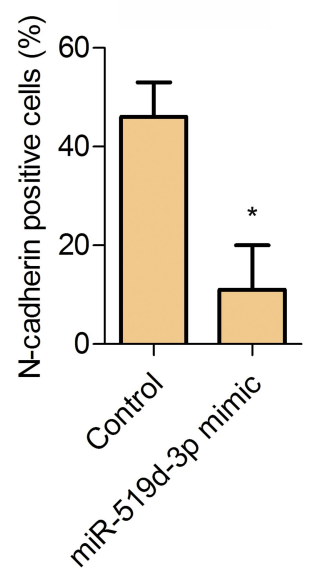

Figure 5 miR-519d-3p affects tumor formation in vivo. (A) Tumor tissue. (B) Tumor volume. (C) Tumor weight. (D) Immunohistochemistry typical images of Ki67 and $\mathrm{N}$-cadherin in tumor tissue. (E) Percentage of Ki67 positive cells. (F) Percentage of $\mathrm{N}$-cadherin positive cells. Data are expressed as mean $\pm \mathrm{SD}$ of three independent experiments $(* P<0.05$ vs control). 
a new research direction. Rosano et $\mathrm{al}^{28}$ revealed that miR$29 a-3 p$ can identify patients with colorectal cancer who are responding to anti-angiogenic therapy. Zhang et $\mathrm{al}^{29}$ reported that miR-34a played an anti-tumor role by inhibiting the expression of VEGF in colorectal cancer. Double luciferase reporter assay revealed that miR-519d$3 p$ could inhibit the fluorescence intensity of VEGF 3'UTR-wt vector significantly, but not VEGF 3'-UTR-mut vector.

Compared with the pcDNA-VEGF transfected group, the invasive cells number and colony forming rate were reduced significantly in the co-transfected group, however, the apoptosis rate was significantly enhanced. These findings suggest that miR-519d-3p inhibits the proliferation, invasion, and promotes apoptosis of lung cancer cells by targeting VEGF.

The PI3K/AKT pathway plays an important role in cancer stem cells (CSCs), including maintaining the ability of colony formation and proliferation. ${ }^{30}$ In addition, targeting the PI3K/AKT pathway could extremely decrease bulk tumor burden and retard metabolism of CSCs. ${ }^{31,32}$ Wang et $\mathrm{al}^{33}$ found that curcumin suppressed cell invasion and migration of NSCLC by elevating miR206 expression which further inhibited PI3K/AKT/ mTOR pathway activation. p38MARK is also involved in the regulation of NSCLC. Boueroy et $\mathrm{al}^{34}$ found that aurisin A could inhibit migration of cancer cells and decrease the expression of epidermal growth factor receptor (EGFR) and phosphorylated p38 (pp38). Xu et $\mathrm{al}^{35}$ reported that TGFßRII knockdown can inhibit cell proliferation, invasion, and induce apoptosis by inhibiting PI3K/Akt and p38 MAPK pathway, and inhibit MMPs and VEGF expression in A549 cells. In the present study, the ratios of p-P38MAPK/P38MAPK, $\mathrm{p}-\mathrm{PI} 3 \mathrm{~K} / \mathrm{PI} 3 \mathrm{~K}$, and $\mathrm{p}-\mathrm{AKT} / \mathrm{AKT}$ in the co-transfection group were reduced significantly compared with the pcDNA-VEGF transfection group. These data indicated that miR-519d-3p inhibits the P38 and PI3K/AKT pathway by targeting VEGF.

\section{Conclusion}

Overexpression of miR-519d-3p inhibits P38 and PI3K/ AKT pathway to attenuate the progress of lung cancer via targeting VEGFA. In vivo, compared with the control group, the miR-519d-3p group had smaller tumor volume and lighter weight. The results were consistent with the results of experiments in vitro. Therefore, miR-519d-3p might be a promising therapy of lung cancer.

\section{Abbreviations}

NSCLC, non-small cell lung cancer; SCLC, small cell lung cancer; miRNAs, microRNAs; VEGF, vascular endothelial growth factor; VEGFA, vascular endothelial growth factor A; EGF, epidermal growth factor; HIF-1 $\alpha$, hypoxia inducible factor- $1 \alpha$; PBS, phosphate-buffered saline; WT, wild-type; MUT, mutant type; IHC, immunohistochemistry; CRC, colorectal cancer; CSCs, cancer stem cells; EGFR, epidermal growth factor receptor.

\section{Author Contributions}

Guangzhao Zhang and Jiahui Du participated in the design of the study, Guangzhao Zhang, Yanlei Hu, WuyingYuan, and Hongli Qiu performed the experiments, Guangzhao Zhang, Yanlei $\mathrm{Hu}$, and Haifeng Yu analyzed the data. Guangzhao Zhang and Yanlei $\mathrm{Hu}$ wrote the manuscript. Guangzhao Zhang conceived and designed the study, analyzed the data, and participated in the preparation of the manuscript. All authors made a significant contribution to the work reported, whether that is in the conception, study design, execution, acquisition of data, analysis and interpretation, or in all these areas; took part in drafting, revising or critically reviewing the article; gave final approval of the version to be published; have agreed on the journal to which the article has been submitted; and agree to be accountable for all aspects of the work.

\section{Disclosure}

The authors report no conflicts of interest in this work.

\section{References}

1. Siegel RL, Miller KD, Jemal A. Cancer statistics, 2016. CA Cancer J Clin. 2016;66(1):7-30.

2. Inamura K, Ishikawa Y. Lung cancer progression and metastasis from the prognostic point of view. Clin Exp Metastasis. 2010;27 (6):389-397. doi:10.1007/s10585-010-9313-4

3. Gridelli C, Rossi A, Carbone DP, et al. Non-small-cell lung cancer. Nat Rev Dis Primers. 2015;1:15009. doi:10.1038/nrdp.2015.9

4. Molina JR, Yang P, Cassivi SD, Schild SE, Adjei AA. Non-small cell lung cancer: epidemiology, risk factors, treatment, and survivorship. Mayo Clinic Proc. 2008;83(5):584-594. doi:10.1016/S0025-6196(11) 60735-0

5. Yuan M, Huang LL, Chen JH, Wu J, Xu Q. The emerging treatment landscape of targeted therapy in non-small-cell lung cancer. Signal Transduction Targeted Ther. 2019;4:61. doi:10.1038/s41392-0190099-9

6. Hembrom AA, Srivastava S, Garg I, Kumar B. MicroRNAs in venous thrombo-embolism. Clin Chim Acta. 2020;504:66-72. doi:10.1016/j. cca.2020.01.034

7. Lodish HF, Zhou B, Liu G, Chen CZ. Micromanagement of the immune system by microRNAs. Nat Rev Immunol. 2008;8 (2):120-130. 
8. Wang Y, Blelloch R. Cell cycle regulation by MicroRNAs in embryonic stem cells. Cancer Res. 2009;69(10):4093-4096. doi:10.1158/ 0008-5472.CAN-09-0309

9. Abdelmohsen K, Kim MM, Srikantan S, et al. miR-519 suppresses tumor growth by reducing HuR levels. Cell Cycle (Georgetown, Tex). 2010;9(7):1354-1359. doi:10.4161/cc.9.7.11164

10. Pal JK, Ray SS, Pal SK. Identifying relevant group of miRNAs in cancer using fuzzy mutual information. Med Biol Eng Comput. 2016;54(4):701-710. doi:10.1007/s11517-015-1360-1

11. Cheng Z, Hou S, Wu Y, et al. LINC01419 promotes cell proliferation and metastasis in lung adenocarcinoma via sponging miR-519b-3p to up-regulate RCCD1. Biochem Biophys Res Commun. 2019;520 (1):107-114. doi:10.1016/j.bbrc.2019.09.090

12. Lin CY, Cho CF, Bai ST, et al. ADAM9 promotes lung cancer progression through vascular remodeling by VEGFA, ANGPT2, and PLAT. Sci Rep. 2017;7(1):15108.

13. Shinkaruk S, Bayle M, Laïn G, Déléris G. Vascular endothelial cell growth factor (VEGF), an emerging target for cancer chemotherapy. Curr Med Chem Anticancer Agents. 2003;3(2):95-117. doi:10.2174/ 1568011033353452

14. Yang J, Yang D, Sun Y, et al. Genetic amplification of the vascular endothelial growth factor (VEGF) pathway genes, including VEGFA, in human osteosarcoma. Cancer. 2011;117(21):4925-4938. doi:10.10 02/cncr.26116

15. Meder L, Schuldt P, Thelen M. Combined VEGF and PD-L1 blockade displays synergistic treatment effects in an autochthonous mouse model of small cell lung cancer. Cancer Res. 2018;78(15):4270-4281.

16. Sun X, Meng L, Qiao W, et al. Vascular endothelial growth factor A/ Vascular endothelial growth factor receptor 2 axis promotes human dental pulp stem cell migration via the FAK/PI3K/Akt and p38 MAPK signalling pathways. Int Endod J. 2019;52(12):1691-1703. doi:10.1111/iej.13179

17. Ye X, Lv H. MicroRNA-519d-3p inhibits cell proliferation and migration by targeting TROAP in colorectal cancer. Biomed Pharmacother/Biomedicine \& Pharmacotherapy. 2018;105:879-886. doi:10.1016/j.biopha.2018.04.114

18. Zhang Y, Guo X, Xiong L, et al. MicroRNA-101 suppresses SOX9-dependent tumorigenicity and promotes favorable prognosis of human hepatocellular carcinoma. FEBS Lett. 2012;586 (24):4362-4370. doi:10.1016/j.febslet.2012.10.053

19. McGuire A, Brown JA, Malone C, McLaughlin R, Kerin MJ. Effects of age on the detection and management of breast cancer. Cancers. 2015;7(2):908-929. doi:10.3390/cancers7020815

20. Gao L, Mei S, Zhang S, et al. Cardio-renal exosomes in myocardial infarction serum regulate proangiogenic paracrine signaling in adipose mesenchymal stem cells. Theranostics. 2020;10(3):1060-1073. doi:10.7150/thno.37678

21. Zhang Y, Xu H. Serum exosomal miR-378 upregulation is associated with poor prognosis in non-small-cell lung cancer patients. J Clin Lab Anal. 2020; 23237.

22. Huang W, Yan Y, Liu Y, et al. Exosomes with low miR-34c-3p expression promote invasion and migration of non-small cell lung cancer by upregulating integrin $\alpha 2 \beta 1$. Signal Transduction Targeted Ther. 2020;5(1):39. doi:10.1038/s41392-020-0133-y

OncoTargets and Therapy

\section{Publish your work in this journal}

OncoTargets and Therapy is an international, peer-reviewed, open access journal focusing on the pathological basis of all cancers, potential targets for therapy and treatment protocols employed to improve the management of cancer patients. The journal also focuses on the impact of management programs and new therapeutic

Submit your manuscript here: https://www.dovepress.com/oncotargets-and-therapy-journal
23. Li Y, Yin Z, Fan J, Zhang S, Yang W. The roles of exosomal miRNAs and $\operatorname{lncRNAs}$ in lung diseases. Signal Transduction Targeted Ther. 2019;4:47. doi:10.1038/s41392-019-0080-7

24. Ramjiawan RR, Griffioen AW, Duda DG. Anti-angiogenesis for cancer revisited: is there a role for combinations with immunotherapy? Angiogenesis. 2017;20(2):185-204.

25. Ma L, Li J. MicroRNA-519d-3p inhibits cell proliferation and cell cycle G1/S transition in glioma by targeting CCND1. Biosci Biotechnol Biochem. 2020;84(2):297-304. doi:10.1080/09168451. 2019.1682510

26. Liang J, Liu Y, Zhang L, Tan J, Li E, Li F. Overexpression of microRNA-519d-3p suppressed the growth of pancreatic cancer cells by inhibiting ribosomal protein S15A-mediated Wnt/beta-catenin signaling. Chem Biol Interact. 2019;304:1-9. doi:10.1016/j. cbi.2019.02.026

27. Qin L, Zhong M, Adah D, et al. A novel tumour suppressor IncRNA F630028010Rik inhibits lung cancer angiogenesis by regulating miR-223-3p. J Cell Mol Med. 2020;24(6):3549-3559. doi:10.1111/ jcmm. 15044

28. Rosano S, Cora D, Parab S, et al. A Regulatory microRNA Network Controls Endothelial Cell Phenotypic Switch During Sprouting Angiogenesis. 2020:9.

29. Zhang D, Qiu X, Li J, Zheng S, Li L, Zhao H. TGF-beta secreted by tumor-associated macrophages promotes proliferation and invasion of colorectal cancer via miR-34a-VEGF axis. Cell Cycle (Georgetown, Tex). 2018;17(24):2766-2778. doi:10.1080/15384101.2018.1556064

30. Zhou J, Wulfkuhle J, Zhang H, et al. Activation of the PTEN/mTOR/ STAT3 pathway in breast cancer stem-like cells is required for viability and maintenance. Proc Natl Acad Sci U S A. 2007;104 (41):16158-16163. doi:10.1073/pnas.0702596104

31. Marhold M, Tomasich E, El-Gazzar A, et al. HIF1alpha regulates mTOR signaling and viability of prostate cancer stem cells. Mol Cancer Res. 2015;13(3):556-564. doi:10.1158/1541-7786.MCR-140153-T

32. Kolev VN, Wright QG, Vidal CM, et al. PI3K/mTOR dual inhibitor VS-5584 preferentially targets cancer stem cells. Cancer Res. 2015;75(2):446-455. doi:10.1158/0008-5472.CAN-14-1223

33. Wang N, Feng T, Liu X, Liu Q. Curcumin inhibits migration and invasion of non-small cell lung cancer cells through up-regulation of miR-206 and suppression of PI3K/AKT/mTOR signaling pathway. Acta Pharmaceutica (Zagreb, Croatia). 2020;70(3):399-409. doi:10.2478/acph-2020-0029

34. Boueroy P, Boonmars T, Kanokmedhakul S, Chareonsudjai S, Lekphrom R, Srichangwang S. Promising anticancer effect of Aurisin a against the human lung cancer A549 cell line. Asian Pac $J$ Cancer Prev. 2020;21(1):49-54. doi:10.31557/APJCP.2020.21.1.49

35. Xu CC, Wu LM, Sun W, Zhang N, Chen WS, Fu XN. Effects of TGF-beta signaling blockade on human A549 lung adenocarcinoma cell lines. Mol Med Rep. 2011;4(5):1007-1015.

agents and protocols on patient perspectives such as quality of life, adherence and satisfaction. The manuscript management system is completely online and includes a very quick and fair peer-review system, which is all easy to use. Visit http://www.dovepress.com/ testimonials.php to read real quotes from published authors. 